\title{
$\gamma$-ray assisted synthesis of silver nanoparticles in chitosan solution and the antibacterial properties
}

\begin{abstract}
In the present study, chitosan had been utilized as a ñgreenò stabilizing agent for the synthesis of spherical silver nanoparticles in the range of $5 \mathrm{i} 30 \mathrm{~nm}$ depending on the percentage of chitosan used $(0.1,0.5,1.0$ and $2.0 \mathrm{wt} \%)$ under J-irradiation. X-ray diffractometer identified the nanoparticles as pure silver having face-centered cubic phase. Ultravioletï visible spectra exhibited the influence of $\mathcal{J}$-irradiation total absorbed dose and chitosan concentration on the yield of silver nanoparticles. The antibacterial properties of the silver nanoparticles were tested against Methicillin-resistant Staphylococcus aureus (MRSA) (gram-positive) and Aeromonas hydrophila (gram-negative) bacteria. This work provides a simple and ñgreenò method for the synthesis of highly stable silver nanoparticles in aqueous solution with good antibacterial property.
\end{abstract}

Keyword: Nanostructures; Electron microscopy; J-ray irradiation; Chitosan; Silver 